\title{
Restoration methods for multi-service optical networks
}

\author{
Admela Jukan and Arnold Monitzer \\ Vienna University of Technology \\ Institute of Communication Networks \\ Gusshausstrasse 25/388, A-1040 Vienna, Austria \\ Tel. +43-1-58801-3993 Fax +43-01-5870583 \\ e-mails: admela.jukan@tuwien.ac.atarnold.monitzer@imladris.ikn.tuwien.ac.at
}

\begin{abstract}
In this paper, we apply different restoration strategies for different optical network services in multi-service optical WDM networks according to the user requirements and availability of network resources. For that purpose optical network services provided to the user networks like SDH or ATM are classified according to the quality degree and restorability required. In particular, the restoration means dynamical reestablishing of circuits, which are generally dynamically or statically assigned. The numerical results for networks with a random restorable topology and four classes of service quality degree as well as for service-specific restoration methods are presented and studied on their applicability.
\end{abstract}

\section{Keywords}

Optical network service, WDM networks, QoS-routing, restorability, blocking probability 


\section{Part One: Routing algorithms for optical networks}

\section{INTRODUCTION}

One of the key parameters of interest for client networks like SDH (Synchronous Digital Hierarchy) or ATM (Asynchronous Transfer Mode) is the service restorability in optical networks. This issue is even more important in case of optical wavelength division multiplexing (WDM) networks where a single fibre is carrying enormous amount of data and a fibre break has an massive impact on carried traffic. The restoration, as discussed here, refers to attempt to re-establish a connection in optical networks, which is being affected by a signal degrade or by the complete loss. Optical network service restorability (ONSR) relates to the efficiency of the restoration method applied. An optical network service (ONS) is defined as any kind of handling of optical signals originating from optical network clients [1]. Like other quality attributes (transmission, access, ...), the ONSR also varies with performance objectives of each single client network. High restorability may be required for clients handling real-time data, while computer communications for other data may tolerate higher connection blocking probabilities or long restoration times. We do not consider any time sensitive attributes of restoration, i.e. we assume that the restoration time is negligible.

Since the Quality-of-Service (QoS)-guarantee is the main issue for integration of optical networks into the existing network infrastructure, the optical network services are here classified according to the quality degree (QoS-guarantee) and restorability required. By using the QoS-routing method to set-up connections, a route capable of providing all necessary QoS-performances for transmission, routing, network management, economic efficiency and restoration is selected for every connection request to the optical layer [1]. We assume that restoration is needed each time the QoS is degraded. The blocking probability is here defined as probability that for a certain service call the required restorability and hence QoS cannot be provided.

\section{RESTORATION METHODS}

The original path that is established at the connection set-up is called the working path. In case of a fibre cut a network has to find an alternative temporary path, a restoration path, for all the affected services of the damaged link. If a restoration path is pre-reserved either exclusively for a connection or by sharing, it is also called a spare path.

In this paper, we distinguish between two restoration schemes depending on restoration endpoints of a connection. One is the restoration between the working path-terminating nodes of the failed path, path-restoration (PR), the other is the restoration between the terminating nodes of a failed link, link-restoration (LR). 
In both of these methods either dynamic- or static (pre-planned) restoration techniques can be applied [2]. With dynamic restoration techniques the spare entities in the case of link failure are dynamically searched. In this case the complex routing algorithm must be applied. The main benefits of dynamic restoration methods are robustness to multiple link failures and a simple a posteriori network expansion. Static restoration techniques reserve spare entities for a connection at the moment of a connection set-up. Since no routing is necessary at the moment of failure, this technique is faster than dynamic restoration. However, the network utilisation is diminishing, because of large number of spare resources reserved.

In this paper, for every existing service a specific restoration method for link failures is assigned. Dynamic Link Restoration (DLR) establishes spare paths between link-terminating nodes of a failed link. For all affected services using a failed link a connection for restoration must be found. For Dynamic Path Restoration (DPR) restoration paths between pathterminating nodes of all affected paths must be found. The number of optical paths to be restored is directly proportional to the traffic on a failed link. Static LR (SLR) is based on preplanned 1:1 spare links that are assigned at the connection set-up. This is supposed to be necessary only for very limited number of services requiring extremely high restorability. Because of the large waste of capacities, Static PR (SPR), based on pre-planned 1:1 spare paths is preferred. It uses the link disjoint paths algorithm, where network graph transforms for path/link-terminating nodes are applied. This algorithm will be explained in more details in the next section.

As a trade-off between fair network utilisation and restorability the methods Shared Static $L R$ (SSLR) and Shared Static PR (SSPR) can be used. The SSLR-method means that a certain wavelength is assigned as a spare for more than one spare path on a link. If a failure occurs, only one spare path can be established per shared wavelength. The SSPR-methods means that the resources used for a certain spare path should be preferably used when assigning another spare paths.

\section{DEFINITION OF RESTORABILITY}

The restoration ratio or restorability of a service $S_{i}$ is the measure of efficiency of a restoration method. The restorability, $\boldsymbol{r}_{i}$, $(i$ indicates the service quality degree) is here defined as:

$$
r_{i}=\frac{\text { successfully restored services } \mathrm{S}_{\mathrm{i}}}{\text { number of attempts to restore a service } \mathrm{S}_{\mathrm{i}}}
$$

For restoration purposes it will be important to find two shortest link-disjoint paths between two nodes. The motivation to find an algorithm that provides link disjoint paths is explained 


\section{Part One: Routing algorithms for optical networks}

with Figure 1. In Figure 1a, the spare path is completely separated from the working one. However, as furthermore shown in Figure 1b, if at least one link of the affected path use the same duct as a link of the working path, the recovery of link failure between the nodes 3 and 7 is not possible. This comes from the fact that mostly all fibres that use the same duct are damaged in case of a fibre cut. For that purpose, it is advantageous to find link disjoint paths.

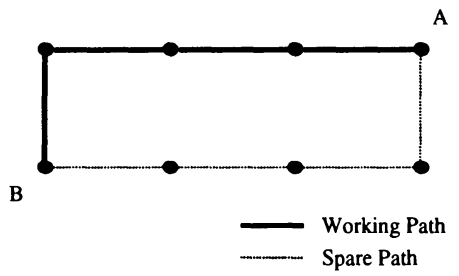

(a)

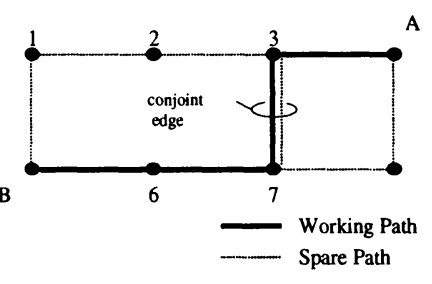

(b)

Figure 1 An example where working and spare path are two link disjoint (a) or conjoint (b) paths

It is not always possible to find a link disjoint path for a certain network topology. For that purpose, we first have to test the network topology, if a link disjoint path exists. This is strongly related to the definition of reliable networks. The fundamental requirement for network reliability can be expressed as the condition that any two subparts of the graph must be connected by at least two links [3]. This is the guarantee that in the case of a single link failure the network remains connected and restoration paths can be established along alternative physical paths. For that reason the nodal degree for every node must be greater or equal 2.

Note that the condition must be modified for survivability, if a node-failure occurs. When designing survivable networks we postulate the following:

Any two subparts of a graph must be connected by at least two disjoint links, and, if there is more than one node in a sub-graph, at least two links must originate from different nodes.

For that purpose, not only link disjoint path algorithms, but also node disjoint path algorithms are necessary. According to that, we can say that a bi-directional bus-topology represents a non-survivable graph. On the other side, a bi-directional ring-topology is survivable for link and node failures.

Now the link disjoint path algorithm (LDPA) is presented in more detail. Let us assume that for a pre-planned restoration technique it is necessary to find a link-disjoint path for the 
working path in the network $G(V, E)$, where $V=\left\{v_{l}, v_{2}, . ., v_{N}\right\}$ is representing $N$ nodes and $E=\left\{e_{1}, e_{2}, . ., e_{L}\right\}$ stays for $L$ links.

Assume that the algorithm is shown for the path-terminating nodes src (source) and dest (destination). The routing algorithm routing(src, dest, G), used here is based on routing with QoS, where for every service call a path capable of providing a QoS guarantee is chosen. The services are classified according to the quality degree to be provided for them. As a shortest path algorithm we use the Dijkstra's shortest path algorithm [4].

In Figure 2 it is shown how the link-disjoint path algorithm is accomplished. First a shortest path from node $s r c$ to node dest, called path $=$ routing $(s r c$, dest, G), is found. The connection path $h_{l}$ between the source and destination node will be established for the connection set-up in a common way. Then in the next step all links of the graph $\mathrm{G}$ between the intermediate nodes of the path ${ }_{1}$ are removed, so that conjoint links are not longer available. Now the graph has to be transformed into a graph $G^{\prime}, G^{\prime}=G \backslash\left\{e_{i}\right\}, \forall e_{i} \in$ path $_{1}$. For the transformed path $G^{\prime}$, the second link disjoint shortest path path ${ }_{2}=$ routing $\left(s r c\right.$, dest, $\left.G^{\prime}\right)$ must be found. With the last step, the path path $_{2}$ is finally the link-disjoint path.

The same algorithm can be applied for finding the restoration path for a one link of a working path, so that the path-terminating nodes $s r c$ and dest are replaced by the link-terminating nodes.

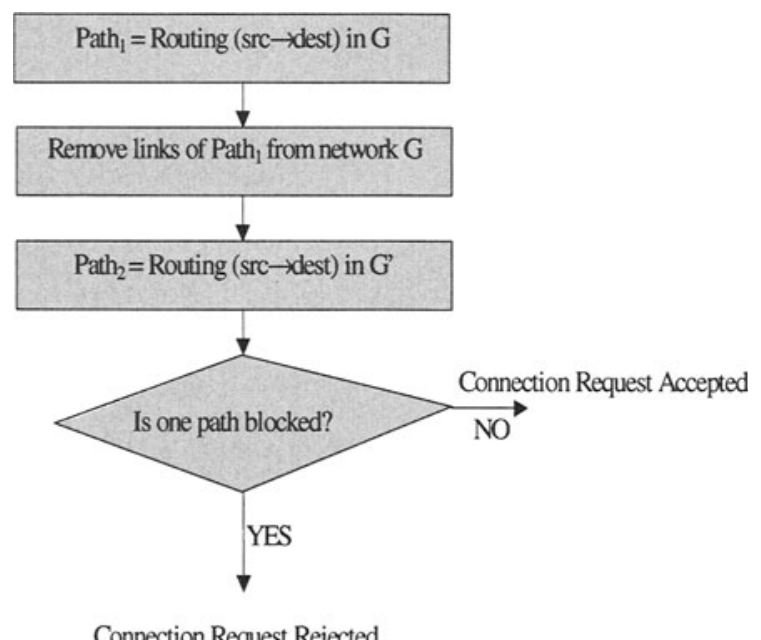

Figure 2 LDP-algorithm

\section{PERFORMANCE STUDY AND SIMULATION RESULTS}

For the numerical examples shown in this section, we use the basic assumptions of circuit switching networks: 


\section{Part One: Routing algorithms for optical networks}

- Poisson service request arrivals normalised to the mean call duration (a call stands for a connection request),

- exponentially distributed call duration times with unit mean,

- no queuing of calls,

- uniform traffic distribution.

The following assumptions for example network topologies are made:

- network load is defined for the entire network as the ratio between arrival- and duration rates,

- restorable random network topology is studied: randomly generated network for a given number of nodes and connectivity (30 nodes, connectivity 0.31 , minimal nodal degree 2 ),

- equal number of wavelengths is assumed per links for the entire network under consideration,

- user calls for connections of a certain quality are randomly generated,

- all results are obtained with a confidence level of $95 \%$,

- Dijkstra shortest path algorithm is used.

For QoS-routing we assume the following:

- four different service classes $\left(S_{0}, S_{1}, S_{2}, S_{3}\right)$,

- with 8 wavelengths per link, the following primary wavelength sets are taken: $W_{\text {prim }}\left[S_{0}\right]=\left\{\lambda_{1}, \lambda_{2}\right\}, W_{\text {prim }}\left[S_{1}\right]=\left\{\lambda_{3}, \lambda_{4}\right\}, W_{\text {prim }}\left[S_{2}\right]=\left\{\lambda_{5}, \lambda_{6}\right\}$, and $W_{\text {prim }}\left[S_{3}\right]=\left\{\lambda_{7}, \lambda_{8}\right\}$,

- wavelengths reserved for lower index (or lower quality) services are generally allowed to use the wavelength of higher-index services (higher quality),

- quality requested from the user side can only be provided by a service of the same or higher quality

Assumptions regarding restoration:

- Poisson process is used as negative exponentially distributed inter-arrival times for link failures (arrival rate is taken as 0.002 time units),

- repair time is deterministic ( 0 time units, i.e. no any new call is affected by a link failure),

- $\quad L D P A$ is used for static restoration methods.

Other parameters will be particularly given.

In Figure 3 a comparison between the degrees of restorability of all introduced methods DPR,

DLR, SPR, SLR, SSPR and SSLR in case where no service classification is provided is given. This is shown for a wavelength-shifted network. The SPR and the SLR are both providing a 
$100 \%$ restorability (both graphs coincide), since we are considering only the link failures. For higher network loads, the DPR shows better restorability with respect to DLR. This is because by establishing many paths between the link-terminating nodes, the blocking probability increases, with respect to the case that many different path-terminating nodes have to be established. If the load is kept low, the DLR and DPR are generally providing nearly the same restorability, because the both methods are the dynamic methods, and directly proportional to the probability of connection accommodation (1-blocking probability).

In Figure 4 the restoration methods DPR and SSPR are compared for the networks with and without wavelength shifting. The benefits in blocking probability for wavelength shifted networks become significant with the increased network size [1]. The same is valid for restorability. Higher restorability is provided by the dynamic methods in a wavelength shifted network. However, the results with the method SSPR significantly differ. Here, the networks without wavelength shifting show better restorability. This comes from the wavelength assignment of a wavelength for sharing. For a wavelength continuous network fewer resources are generally shared since the paths are longer, i.e. the probability that a wavelength can be shared is smaller. This is not the case with a wavelength-shifting network.

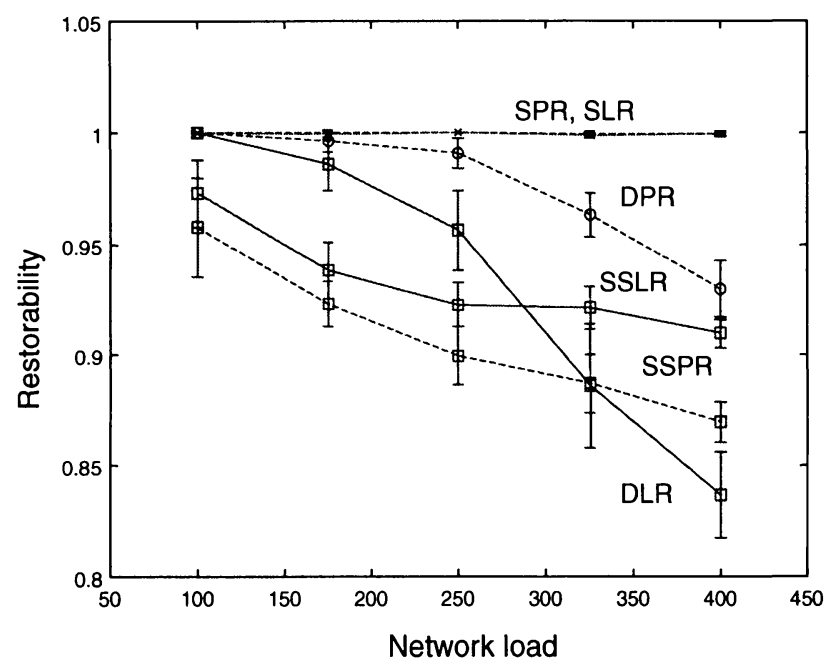

Figure 3 A comparison between different restoration methods for our example network (dashed line- path methods, full line-link methods). 


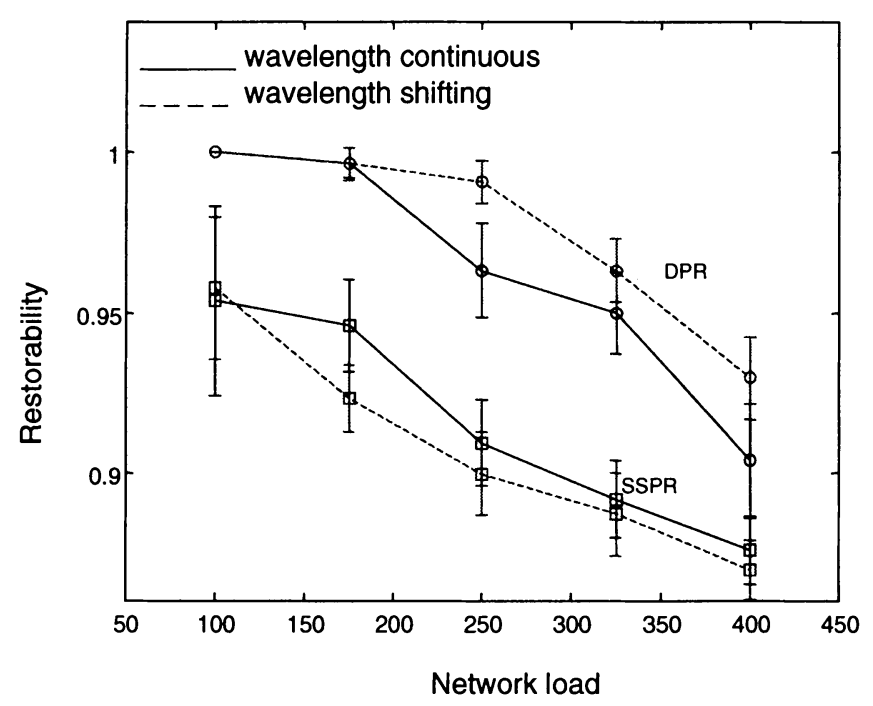

Figure 4 Restorability for DPR and SSPR methods for the example network with and without wavelength shifting.

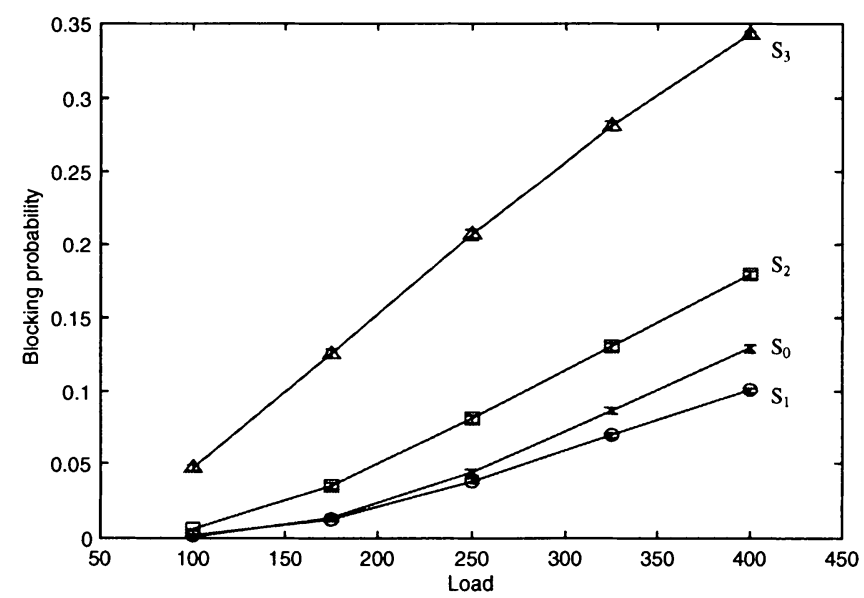

Figure 5 Blocking probability of four services in 30 nodes WDM transport network (wavelength shifted, $100 \%$ link failures, (x) $S_{0}$ with SPR, (o) $S_{1}$ with no restoration, ( $\square$ ), $S_{2}$ with DLR, $(\Lambda)$ service $S_{3}$ with DLR)

For the same network we now assume 4 different service classes, where the following restoration methods are assumed: SPR for $S_{0}$, no restoration for $S_{1}$, DLR for $S_{2}$, and DLR $S_{3}$. This is shown in Figure 5 and Figure 6. It can be expected that the blocking probability of 
service $S_{0}$ would be the lowest, because all free wavelengths of $\lambda_{1}-\lambda_{2}$ are available for this service [1]. Since the SPR method is used for this service, blocking probability increases very much and exceeds blocking probability of service $S_{I}$. Blocking probability of services $S_{1}-S_{3}$ will not be changed with respect where no failures happen, because for dynamic methods no spare entities have to be allocated.

In Figure 6, the restorability per service is shown. The service $S_{0}$ provides $100 \%$ restorability, while the restorability of service $S_{l}$ is zero for any network load (for lower network loads, the uncertainty of results increases, since no path will be affected during a sample period of confidence). It can be seen that service $S_{2}$ is assigned to a better restorability than service $S_{3}$, because more wavelengths can be available to the service $S_{2}$.

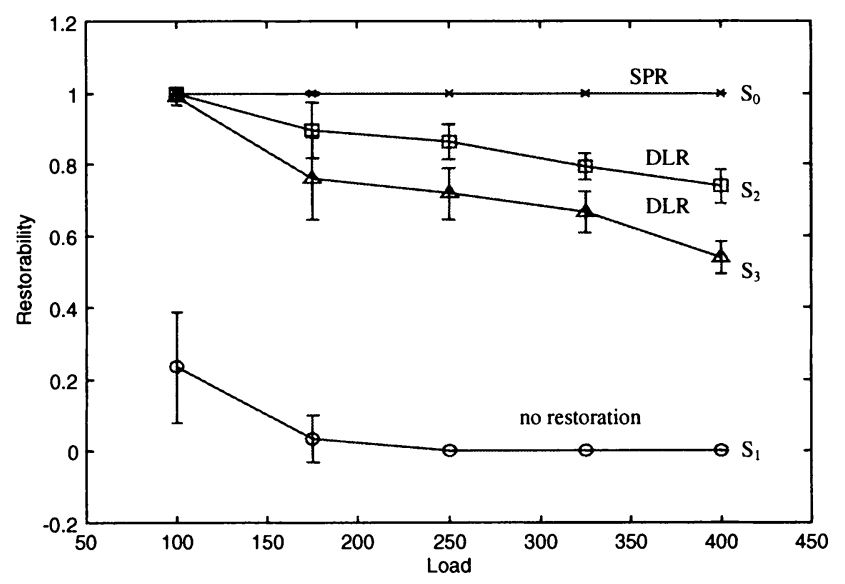

Figure 6 Restorability for different services with different restoration methods assigned (the same assumptions as in Figure 5).

\section{Conclusion}

In this work, different restoration methods are applied to different services and connections in optical networks. This comes naturally from the fact that the client networks are having different requirements on the QoS and hence on the restorability for a connection. The restorability and network utilisation efficiency are two opposite conditions to fulfil and a trade-off between service blocking probability and appropriate restorability must be found. We showed that an important issue for the future study is the performance comparison 
12 Part One: Routing algorithms for optical networks

between networks with and without wavelength shifting, which use service-specific restoration methods.

\section{References:}

1. A. Jukan, H.R. van As: Quality-of-service routing in optical networks, Proceedings on ECOC'97, Session We2D, pp. 160-163, 1997

2. T.-H. Wu: Fiber Network Survivability, Artech House Inc, Boston-London, 1992.

3. S. Baroni, P. Bayvel: Wavelength Requirements in Arbitrarily Connected WavelengthRouted Optical Networks, Journal of Lightwave Technology, vol. 15, No.2, Feb. 1997.

4. A. Kershenbaum: Telecommunications Network Design Algorithms, McGraw-Hill, Inc, 1993. 